\title{
Fault Analysis for Power Network Equipment Based On Association Rule Clustering Algorithm
}

\author{
Zhang $\mathrm{Hu}^{1}$, Li Feng $^{1}$ and Wang Hongbin ${ }^{1}$ \\ 1. Electric Power Research Institute of Guangdong Power Grid Co., Ltd., \\ Guangzhou 510000, Guangdong Province, China \\ 13083104@qq.com
}

\begin{abstract}
In order to improve the fault positioning accuracy of distributed generation power distribution network, it puts forward fault positioning method of distr bution network equipment based on blended data association rule data minhing method. Based on fuzzy rough set theory, it studies classification rule mining method on hybric data, through the introduction of the rule to obtain the a generalized threshold of the algorithm, and control the scale and complexity of the obtained rule set, so as to improve the classification efficiency of rough set method of knowledge discovery on the failure data and get the fault positioning feature of distribution network, findlly adopts the support vector machine (SVM) to make fault classification, and tests the performance of the algorithm with simulation experiment. The simulationcesylts show that, this paper can be quickly and accurately to locate fault power section, and the fam positioning accuracy is higher than other fault positioning methods of dustribution network.
\end{abstract}

Keywords: Rough set; fault positioning; Association rule; Power grid equipment; Modal data

\section{Introduction}

With the rapid development of our country economy, and the constant improvement of peopie's liffe quality, the demand for electricity in the production and living is becoming more and more large[1]. Distributed Generation, DG technology has changed the structure and operation mode of distribution network, puts forward higher requirements on fault positioning reliability, so how to improve the fault positioning accuracy of Distributed generation power distribution network, become a hospot in current study of electrical applications [2]. Due to containing distributed generation power distribution network fault positioning has very important practical application value, a lot of experts and scholars have put into the study of the problem, and proposed many effective fault positioning algorithms of containing distributed generation power distribution network [3, 4]. The fault positioning method of current containing distributed generation power distribution network is mainly divided into two categories: one is fault positioning method based on perfect information, another kind is fault positioning method based on the imperfect information [5]. In positioning method based on perfect information, the matrix algorithm is widely studied due to its fast computing speed.

There has no Literature on the analysis and research on rough set rules for blended data mining method. This paper makes systematic research on rule mining algorithm based on fuzzy rough set model, the method leaves out blended attribute of pretreatment process, can be directly analyzed the blended data, and provides support for the classification knowledge of network equipment fault diagnosis data with rough set method. With a good adaptability, this new method can effectively decompose the complex signal into several intrinsic mode features (IMF) can 
highlight the local signal features, can better grasp the original local signal fe atures, using the EMD decomposition method is not only no need of selecting basis function, but also can be decomposed according to the signal characteristics, and can highlight the local features of signal accurately, with very good adaptability as well[11]. In order to improve the fault positioning accuracy of distribution network, aiming at the shortcomings of the current fault positioning method, it puts forward based on the fault positioning method of distribution network based on empirical mode decomposition, and tests the performance of this method with simulation test. The simulation results show that the distribution network fault positioning algorithm can quickly, accurately to locate fault interval.

\section{LERS Rule Mining System Based on Rough Set}

LERS rule mining system based on rough set is the most widely used one of rough set knowledge acquisition system. Algorithm LEM2 is the typical fulle mining algorithm of LERS system, the upper and lower approximation set of Pawlak rough set model as the input of the algorithm, sets certainty rule and possibility rule for inconsistent data sets. LEM2 algorithm has been successfully applied in the knowledge discovery and data mining of fault diagnosis decision Table [4].

\subsection{Rough Set Rule Mining Model}

The basic concept of algorithm LEMM is using attribute value for modeling. For an attribute value, $t=\left(c_{j}, v\right), c_{j} \in G, D \in V$, the block of $t$ is denoted as $[t]$, it is composed by a series of object in the the fry of domain $U$, these objects satisfy $\left\{x \mid x_{j}=v, x \in U\right\}$. Assumed that $B$ is a non-empty lower and upper approximate set of category set, $\mathrm{T}$ is the atribute value pairset of one object, that is set $B$ depends on set $T$ if and only if $\varnothing \neq \equiv[T]=\cap_{t \in T}[t]<B$. Set $T$ is called as the minimum coverage of set $B$, if and only 1 f $B$ depends on $T$, an no other subset $T^{\prime}$ of $T$, satisfies $B$ depends on $T^{\prime}$.Assumed Cis a non-empty minimum set, $\mathbf{C}$ is the local coverage of $B$, if and only if:

1) $\cup_{T \in \mathrm{n}}[T]=B$,

1) $\cup_{T \in \mathrm{n}}[T]=B$,

2) $\mathbf{C}$ is minimum that is no subset $\mathbf{C}^{\prime}$ of $\mathbf{C}$ satisfies condition 1).

Algorithm LEM2 adopts strategy based on priority, each selection has attribute value with the highest priority and add it into the partial minimum coverage. When given a partial coverage, the algorithm transformed into a set of rules set. Rule mining based on rough set method does not need to make changes on model, can combine with the discretization method, attribute reduction method for the knowledge acquisition on fault diagnosis blended data.

\subsection{Measurement of Classification Rule}

Pawlak gave the property of generalized decision rule. For a given decision information system DIS,DK indicates a generalized decision class, where $K$ represents example set related to DK. $p$ represents a basic condition, that is the $(a, v)$ condition attribute-value pair,where $a \in C$ and $v \in V a$, assumed $\Phi=p_{1} \wedge p_{2} \wedge \ldots \wedge p_{n}$ is a conjunctive form of basic condition, $[\Phi]$ represents the coverage of $\Phi$, that is the sample set satisfying all basic conditions of $\Phi,[\Phi]_{K}^{+}=[\Phi] \cap K$ is called as the positive coverage of $\Phi$ on $K,[\Phi]_{K}^{-}=[\Phi] \cap(U \backslash K)$ is called as negative coverage on $K$. 
Definition 1 For a given decision list $D I S, D K$ is a generalized decision class, the classification rule $\mathrm{r}$ can be expressed as:

IF $\Phi$ THEN $D K$,

Or simplified as : $\Phi \rightarrow D_{K}$.

Where, $\Phi$ is the condition of $r$, satisfies $[\Phi]_{K}^{+} \neq \varnothing$, and $D K$ is called as decision part of $r$. If $D K$ is a single decision, rule $\mathrm{r}$ is called as certainty rule, if $D K$ is the association of multiple decisions, then rule $r$ is called as possibility rule.

Definition 2. For a given classification rule $\mathrm{r}, \mathrm{K}$ is the sample set covered by generalized decision $D K$, classification rule $\mathrm{r}$ relative to $\mathrm{K}$ is significant when and only when it meets the following conditions:

(1). Consistency : $[\Phi]_{K}^{-} \neq \varnothing$;

(2). Minimum : if delete any basic condition pi from $\Phi$, and no longer meet with consistent.

Definition 3 For a given classification rule set $\mathrm{R}, D K$ is a generalized decision class, if the description of R on DK satisfied the following condition, then $\mathrm{R}$ can be called as a minimum rule set.

(1). For random $r \in R$ it is significant ;

(2). $\cup_{r \in R}[\Phi]=K$;

(3). Delete any rule $r \in R, R \backslash\{r\}$ no longer Satisfies condition (1) and (2).

Definition 4 For a given decision information system $D I S, \mathrm{r}$ is the classification rule of decision table, $\Phi$ represents condytion of the rule, DK is the decision of the rule, while the support, coverage and reliability of some decision $d i \in V d$ can be respectively defined as:

$$
\begin{aligned}
& \mu_{\text {sup }}(r)=|[\Phi]| /|U| \\
& \mu_{\text {cov }}(r)=[\Phi]|/| D_{K} \mid \\
& \mu_{c e r}\left(r, d_{i}\right)=\left|[\Phi]_{d}^{+}\right| / \mid[\Phi\}
\end{aligned}
$$

It can be known from definition 4, support indicates the samples of support rule in the theory of domain; coverage indicates the proportion of supporting number in the corresponding generalized decision classes; credibility indicates the probability of correctly using the rule for reasoning. After obtaining classification rules, it needs to evaluate the extracted rule. The common method is the recognition rate of test rule on test sample classification, the smaller the error rate is, the better of the rule it is.

\section{Rule Mining Algorithm of Blended Data of Fault Diagnosis on Power Network Equipment}

Pawlak rough set model only operate on the data set containing symbol attribute. The fault diagnosis decision table usually contains heterogeneous attribute. The following will discuss classification rule mining algorithm of blended data on the basis of the fuzzy rough set model.

\subsection{Rule Mining Model Based on Fuzzy Rough Set}

A clear equivalence relation can be generated as the clear division on the theory of domain, and a fuzzy equivalence relation can be generated as the fuzzy partition on the theory of domain. Therefore, attribute values in rough set rule mining algorithm on block definition can be naturally extended to fuzzy equivalence space.

Definition 5 Assumed $t=\left(c_{j}, F\right)$ is the attribute value pair of blended decision information system, then the fuzzy block definition of $t$ is : 


$$
[t]=\int_{x \in U} \mu_{F}\left(x_{j}\right)
$$

Obviously, $[t]$ is fuzzy equivalence class generated by $\mathrm{x}$ on fuzzy equivalence relation $\mathrm{R}$. According to the nature of the fuzzy equivalence relation, $[t]$ is a fuzzy set. This is the primary difference between fuzzy block and clear block. It's easy to see that, as the clear set is the natural extension of the fuzzy set, the definition of fuzzy block is the natural extension to the definition of clear block. If the property is discrete, equivalence relation matrix and equivalence class will be degenerated into block definition of classic algorithm.

Definition 6 Assumed $\mathrm{R}$ is fuzzy equivalence relation, $\mathrm{X}$ is the clear set of object, then the upper, lower approximation set is defined as:

$$
\begin{aligned}
& \underline{R} X=\left\{x \mid[x]_{R} \subseteq X, x \in U\right\} \\
& \bar{R} X=\left\{x \mid[x]_{R} \cap X \neq \varnothing, x \in U\right\}
\end{aligned}
$$

The operation " $\subseteq$ " contained in fuzzy set theory is first proposed by professor Zadeh,called as Zadeh contain. However, this definition is over strict ih practical application. Here, it adopts a week contain operator $\left.\subset_{\alpha}\right)$, that is $\left.\forall x\right\rangle \in U, A \subseteq_{\alpha} B$ if and only if $\max \left(1-\mu_{A}(x), \mu_{B}(x)\right) \geq \alpha$.

Definition 7 Assumed $X$ is a clear set, $T$ is a set of attribute value pair, set $\mathrm{X} \alpha$ approximately depend on set $T$ if and only $i$

$$
\begin{aligned}
& \varnothing \neq[T]=\cap_{t \in T}[t] \subseteq_{\alpha} X, \\
& \text { Where, } A \cap B=\int_{x \in U} \min \left(\mu_{A}(x), \mu_{B}(0)\right) x .
\end{aligned}
$$

Assumed B is a classified porempty lowen upper approximation clear concept set, $T$ is a attribute value pair set. Set $T$ is one approximate minimum coverage of $B$, if and only if $B \alpha$-approximately depend on set Tand no subset $T^{\prime}$ of $T$ can make $B$ satisfies $\alpha$ - approximatel $Q$ depend on $T^{\prime}$

Definition 8 Assumed $\mathcal{C}$ is a nonenty approximate minimum coverage set, that is the set of attribute value paif, B is a classified clear object set on theory of domain. $\mathrm{C}$ is $\mathrm{a}(\mathrm{\alpha}, k)$ of $\mathrm{B}$, approximately local coverage, if and only if the following conditions are satisfied:

Definition 8 Assumed $C_{\text {s }}$ a non-empty approximate minimum coverage set, that is the set of attribute value pair, B is a classified clear object set on theory of domain. $\mathrm{C}$ is a $(\alpha, \mathrm{k})$ of $\mathrm{B}$, approximately local coverage, if and only if the following conditions are satisfied:

1) For any element $T$ of $\mathbf{C}, T$ is a $\alpha$-approximate minimum coverage of $\mathrm{B}$,

2) $\left.H\left(\varphi_{T,}, T\right], B\right) \leq k$,

3) (1. the minimum, that is $C$ has the least element,

$$
\begin{aligned}
& \text { Whre, the dissimilarity degree of two fuzzy sets are defined } \\
& H(A, B)=\frac{|\bar{A} \cap B|+|A \cap \bar{B}|}{|A \cup B|},|\bullet|=\sum_{x \in U} \mu_{\bullet}(x), \bar{A}=\int_{x \in U}\left(1-\mu_{A}(x)\right) / x, H(A, B) \in[0,1] .
\end{aligned}
$$

By definition, $\alpha$ and $\mathrm{k}$ provides new stopping criteria for rule mining algorithm. When $\alpha=0, k=0$, attribute is discrete variable, the stopping criteria is degraded into the same stopping condition as the algorithm LEM2. Because the stopping condition of algorithm LEM2 is too strict, it may extract too detailed classification rules, and make the obtained knowledge overfitting the training data, and running time of the algorithm is therefore increased dramatically. To solve this problem, $\alpha$ and $\mathrm{k}$ are introduced into rule mining algorithm, and allowed the input set partially depend on concept set, which makes local coverage can not contain a small part of the training data. Parameter $\alpha$ and $\mathrm{k}$ play the role of relaxation rule mining 
algorithm, when the data set contains distribution overlapping class or inconsistent objects, this mechanism is very effective.

\subsection{Rule Mining Algorithm of Fuzzy Rough Set on Blended Data}

Algorithm LEM2 uses the heuristic information to extract frequent attribute value to compose the minimum coverage, the same as LEM2, algorithm FRLEM2 according to the definition of score function to select attribute value with the highest score each time to form the approximated minimum coverage, through the parameter $\alpha$ and $\mathrm{k}$ to control the complexity of the generated rule set effectively, and improve the generalization ability to acquire knowledge. Due to the definition of fuzzy block is different from classical block, it needs to redefine the score function of attribute value.

Definition 9 For the given attribute value pair $t$ and fuzzy set $G$, $t$ relative to $G$ score function is defined as:

$\operatorname{Score}(t, G)=|[t] \cap G|$

Assumed $H D I S=\left\{U, C^{s} \cup C^{n} \cup C^{f} \cup\{d\}, V, f\right\}$ is the blended decision table, where $C^{s}$ represents symbolic attribute set, $C^{\prime}$ represents numeric type attribute set, $C^{f}$ represents fuzzy type attribute set, $d$ represents decision attribute. $B$ represents the lower, upper approximation set of some class in training data. $\alpha$ and $k$ are two arithmetic parameters usually set near zero,thefollowing provide the code of classification rule mining algorithm NRLEM2.

Algorithm: FRLEM2 rule mining algorithm based on fuzzy rough set model Input: blended decision taple $H D I S$, a non-enty concept set $\mathrm{B}$, parameter $\alpha$ and $k$.

Output: a $(\alpha, k)$ - approximation locål coverage $\mathrm{C}$;

1: $\mathrm{G} \Leftarrow \mathrm{B}$;

2: $\mathbf{C} \Leftarrow \varnothing$;

3: while $H(\cup(T)[T, B)>\mathrm{k}$ do

$4:$

5:

while $(T-\varnothing)$ or $\left(\operatorname{not}\left([T] \subseteq_{\alpha} B\right)\right)$ do

6:

7:

$t \longleftarrow \arg \max _{t \in T} \operatorname{Score}(t, G)$;

8:

9:

10 :

\section{1 end while}

$T \Leftarrow T \cup\{t\} ;$

$G \Leftarrow[t] \cup G ;$

for $\forall t \in T$ do

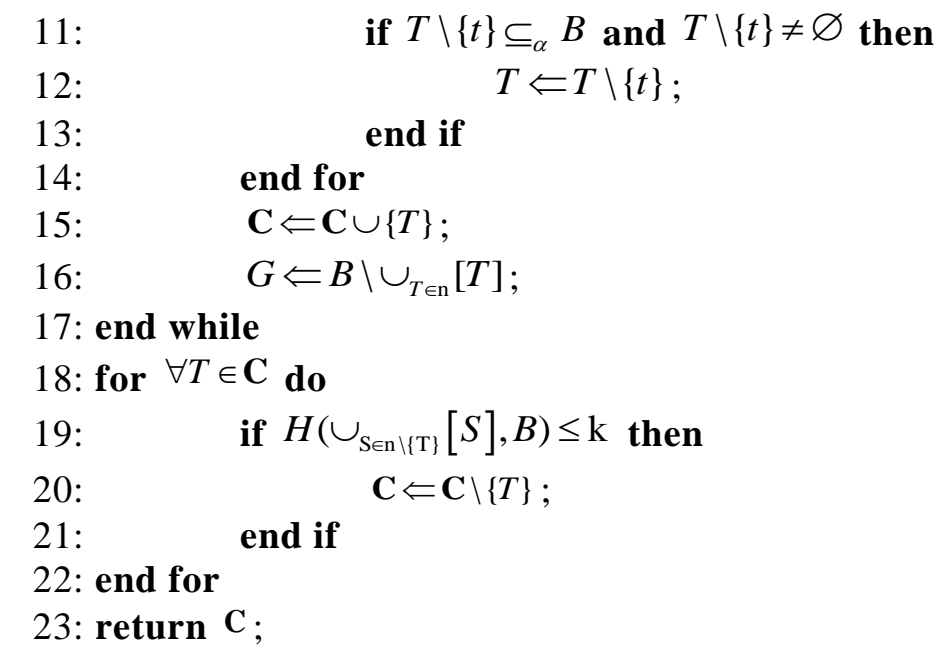


Theorem 1. When $k=\alpha=0$, and attribute are all continuous attribute, classification rule mining algorithm FRLEM2 is degraded into algorithm LEM2.

Testify: if $k=\alpha=0$, and attribute are all continuous type, the following properties of algorithm FRLEM2 are come into existence:

1) The fuzzy equivalence relation is degraded into clear equivalence relation;

2) The definition of $\alpha$ is equivalent to classical dependency;

3) $(\alpha, k)$ - local coverage is equivalent to classical local coverage;

4) Fuzzy set operation is degraded into classic set operation.

It can be known from 1), 2), 3) and 4), the stopping criterion of rule mining process of algorithm FRLEM2 and LEM2 is equivalent, so the theorem comes into existence.

Theorem 1 shows the algorithm LEM2 is the particular case of atgorithm FRLEM2 at $k=0, \alpha=0$, and attribute are all discrete types. In fact, the algonthm FRLEM2 avoids the too strict stopping criterion and the limits of algorithm LEM2 to attribute type. The performance of the algorithm FRLEM2 can also be adjusted by the parameter $\alpha$ and $\mathrm{k}$.

\section{Simulation Experiment}

\subsection{Simulation Environment}

In order to test the rationality and validity of the algorithm in this paper, on the Pentium (R) 4 core $2.8 \mathrm{GHz}, 8 \mathrm{G} \mathrm{RAM}$, Windows 7 operating system, to carry out simulation experiment with MATLAB R2010b. Simulation model is the neutral point un-grounded distribution system, bus bar voltage is $10 \mathrm{KV}$, two distributed powers DG1 and DG2 are $2 \mathrm{MW}$ and 1.5 $\mathrm{MWW}$ respectively.

\subsection{Result Analysis,}

When the start point of fault is $0.005 \mathrm{~s}$, the fault range positioning results of distributed powerdistributionnetwork by using the EMD algorithm are as shown in Table 1. From the analysis of Fable 1, you can get the following conclusions:

When the single-phase grounding fault occurs, compared the maximum value of diff (IMF3) modulus of individual monitoring-station fault on zero sequence current, the fault branch of diff (IMF3) modulus maximum is larger than that of non-fault branch, the largest section of modulus maximum value is the fault power section.

With the mcrease of transition resistance, the diff (IMF3) modulus maximum value shows a trend of decrease, this is because the increase of transition resistance would result in a transient signal weakened. But as long as zero sequence current exists. it must contain the transient information, the overall trend of the signal has not changed, by using EMD decomposition method can extract the transient information, so the size of the transition resistance does not affect the positioning result. 
Table 1. Fault Positioning Result of Containing Distributed Power Distribution Network (0.05s)

\begin{tabular}{ccccccc}
\hline \multirow{2}{*}{ fault point } & \multicolumn{5}{c}{ monitoring point No } & \multirow{2}{*}{ positioning result } \\
\cline { 2 - 6 } F1 & 1 & 2 & 3 & 4 & 5 & \\
\hline \multirow{3}{*}{ F2 } & 1.991 & 7.484 & 0.959 & 2.009 & 2.045 & \\
& 1.192 & 4.574 & 0.447 & 1.317 & 1.064 & fault power section2 \\
& 0.828 & 3.742 & 0.376 & 0.571 & 0.888 & \\
& 0.311 & 0.283 & 0.280 & 1.325 & 0.429 & \\
& 0.204 & 0.143 & 0.107 & 1.091 & 0.256 & fault power section4 \\
& 0.273 & 0.161 & 0.092 & 1.032 & 0.171 & \\
& 0.628 & 0.564 & 0.326 & 0.770 & 2.540 \\
& 0.443 & 0.625 & 0.291 & 0.94 & 2.540 & fault poyver section5 \\
& 0.380 & 0.383 & 0.218 & 0.553 & 1.230
\end{tabular}

When the start point of fault is $0,0 \mathrm{~N}$, the faut range positioning results of distributed power distribution netwo k by using the EMD algorithm are as shown in Table 2. From the analysis of Table 2 , you can get the following conclusions:

(1)After the decomposition of fault zero sequence current at each monitoring point, the maximum values of diff (IMF3 3 modulus are much smaller than that of the Table 1, mainly because the frequency and amplitude of fault zero-sequence transient current are determined by the transient capacitance current, and the amplitude and initialphase Angle are related.

(2)When the failure of maximum voltage phase Angle (0.005 s) occurs, the capacitive carrent will reach the maximum; when a failure occurs near the voltage phase Angle of 0, transient component of capacitive current will be smaller. Although the differen farmure time will affect the size of the amplitude, but the overall trend of signar would not be changed.

(3)The maximum of diff(IMF3) modulus of single-phase grounding fault branch is obviously larger than that of non-fault branch, and positioning results are accurate, the results showed that in different time of single-phase grounding fault occurs, the results of section position of distributed power distribution network with algorithin EMD are not affected by fault point. 
Table 2. Fault Positioning Result of Containing Distributed Power Distribution Network (0.01s)

\begin{tabular}{|c|c|c|c|c|c|c|}
\hline \multirow{2}{*}{ fault point } & \multicolumn{5}{|c|}{ monitoring point No } & \multirow{2}{*}{ positioning result } \\
\hline & 1 & 2 & 3 & 4 & 5 & \\
\hline \multirow{3}{*}{$\mathrm{F} 1$} & 0.287 & 1.124 & 0.104 & 0.298 & 0.341 & \multirow{3}{*}{ fault power section 2} \\
\hline & 0.229 & 1.247 & 0.111 & 0.260 & 0.266 & \\
\hline & 0.190 & 0.942 & 0.107 & 0.294 & 0.172 & \\
\hline \multirow{3}{*}{ F2 } & 0.098 & 0.080 & 0.041 & 0.348 & 0.137 & \multirow{3}{*}{ fault power section 4} \\
\hline & 0.115 & 0.110 & 0.047 & 0.453 & 0.097 & \\
\hline & 0.070 & 0.084 & 0.039 & 0.361 & 0.077 & \\
\hline \multirow{3}{*}{ F3 } & 0.162 & 0.122 & 0.042 & 0.133 & 0.377 & \multirow{3}{*}{ fault pow } \\
\hline & 0.128 & 0.141 & 0.049 & 0.099 & & \\
\hline & 0.109 & 0.150 & 0.050 & 0.132 & & \\
\hline
\end{tabular}

\subsection{Performance Comparison with Other Fault Positioning Method of Power}

\section{Distribution Network}

In order to test the performance of the method in this paper, it selects distribution network fault positioning methods from literature [12] and [13] for comparison, and the single fault and double faults example ate used for simulation experiment. The fault positioning accuracy and pesitioning time are shown in Figure 1 and 2. From Figure 1 and 2, it is known that compared with the contrast method, the ingle fault and double faults positioning accuracy of algorithm for DG distribution network is above $96 \%$, and almost no misjudgment, and fault positioning speed is faster, the simulation results proved that the method can accurately and efficiently solve the fault positioning problem of power distribution network.

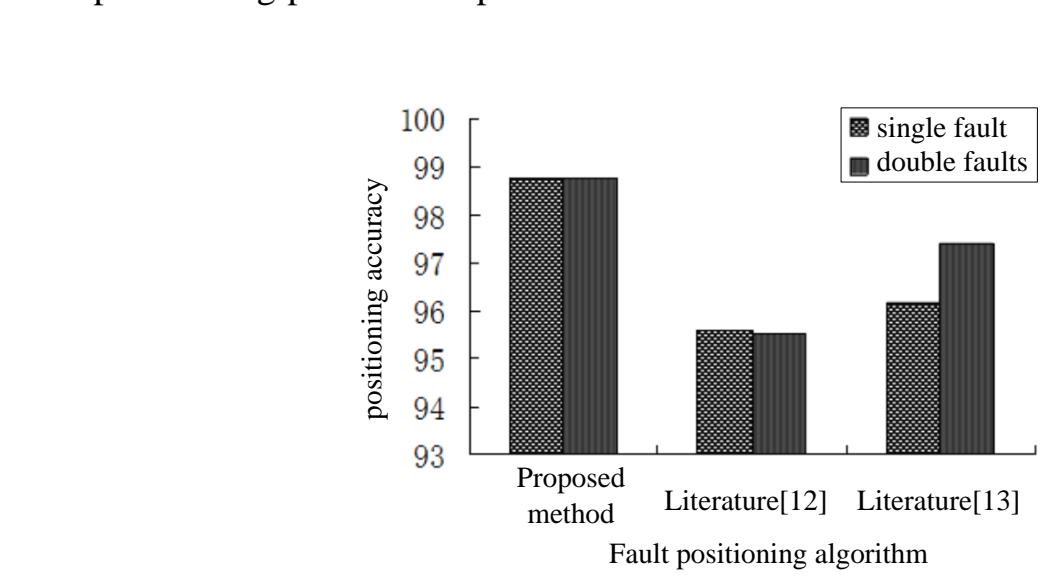

Figure 1. Comparison of Fault Positioning Accuracy of Power Distribution Network with Different Methods 


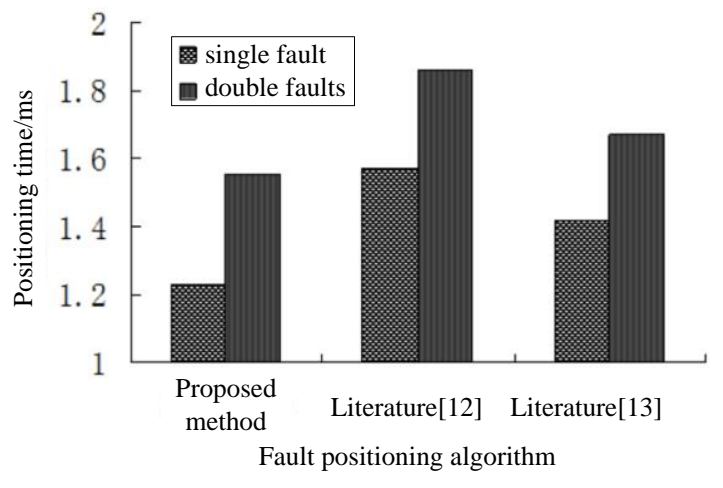

Figure 2. Comparison of Fault Positioning Speed of Power Distribution Network with Different Methods

\section{Conclusion}

In view of the existing fault positioning has the problem of low accuracy, serious misjudgment, this paper puts forward a kind of fault positioning method of grid equipment based on association rule data mining. The simulation results show that the method can make accurate positioning on the fault of containing distributed power distribution network, and can meet the requirements of fault positioning speed of distribution network, has certan practicalapplication value.

\section{References}

[1] Z. Lv, A. Halawani, S. Feng, H. Li and S. Jr Rehman, "Multimodal Hand and Foot Gesture Interaction for Handheld Devices", ACM Transactions on Multimedia Computing, Communications, and Applications (TOMM), vol. 11, no. 1s, Artidle 10, (2014), 19 pages.

[2] K. Leng, W. Shi, J Chen and Z Av, "Designing of a I-shaped less-than-truckload cross-dock: A simulation experiments study', International Journal of Bifurcation and Chaos, (2015).

[3] Y. Lin, J. Yang, Z. Lv, W. Wei and H. Song, "A Self-Assessment Stereo Capture Model Applicable to the Internet of Things", Sersors, (2015).

[4] B. Jie, K. Xu and J. Giu. Analysis and management of the operation and management of power generation units during the low load period", Jiangsu electrical engineering, (2015).

[5] Y. Huang, "Research on transmission network planning based on improved hybrid differential evolution algorithm", Jiangsu electrical engineering, (2015).

[6] M. Shao and D. Yan, "Research and optimization of Nanjing electric power communication transmission network", Jiangsu electric engineering, (2015).

[7] K. Wang "Next generation job management systems for extreme-scale ensemble computing", Proceedings of the 23rd international symposium on High-performance parallel and distributed computing. ACM, (2014).

[8] T. Li, "Distributed Key-Value Store on HPC and Cloud Systems", 2nd Greater Chicago Area System Research Workshop (GCASR), (2013).

[9] Y. Geng, J. Chen, R. Fu, G. Bao and K. Pahlavan, "Enlighten wearable physiological monitoring systems: On-body rf characteristics based human motion classification using a support vector machine, IEEE transactions on mobile computing, vol. 1, no.1, (2015), pp.1-15.

[10] J. He, Y. Geng, F. Liu and C. Xu, "CC-KF: Enhanced TOA Performance in Multipath and NLOS Indoor Extreme Environment”, IEEE Sensor Journal, vol. 14, no. 11, (2014), pp. 3766-3774.

[11] S. Zhou, L. Mi, H. Chen and Y. Geng, "Building detection in Digital surface model", 2013 IEEE International Conference on Imaging Systems and Techniques (IST), (2012).

[12] W. Ou, Z. Lv and Z. Xie, "Spatially Regularized Latent topic Model for Simultaneous object discovery and segmentation", The 2015 IEEE International Conference on Systems, Man and Cybernetics, (SMC2015).

[13] K. Wang, "Using Simulation to Explore Distributed Key-Value Stores for Exascale System Services", 2nd Greater Chicago Area System Research Workshop, (GCASR), (2013).

[14] Y. Wang, Y. Su and G. Agrawal, "A Novel Approach for Approximate Aggregations Over Arrays. In Proceedings of the 27th international conference on scientific and statistical database management, ACM, (2015). 
[15] Z. Lv, A. Halawani, S. Feng, S. ur Rehman and H. Li, “Touch-less Interactive Augmented Reality Game on Vision Based Wearable Device", Personal and Ubiquitous Computing, (2015).

[16] J. Yang, S. He, Y. Lin and Z. Lv, "Multimedia cloud transmission and storage system based on internet of things", Multimedia Tools and Applications, (2016).

[17] Y. Su, "In-situ bitmaps generation and efficient data analysis based on bitmaps", In Proceedings of the 24th International Symposium on High-Performance Parallel and Distributed Computing, ACM, (2015), pp. 61-72.

[18] D. Jiang, X. Ying, Y. Han and Z. Lv, "Collaborative Multi-hop Routing in Cognitive Wireless Networks", Wireless Personal Communications, (2015).

\section{Authors}

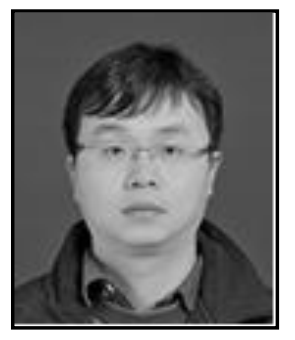

Zhang Hu, He received his M.S. and D.S. degree in automation engineering from Huazhong University of Science and Technology in Wuhan, China. He is currently a Post-Doctor in the Electric Power Research Institute of Guangdong Power Grid Co., Ltd. F His research interest is mainly in the area of Data Mining, Patterm-Recognition, and Smart Grid. He has published several research papers in scholarly journals in the above research-areas and has participated in several projects.

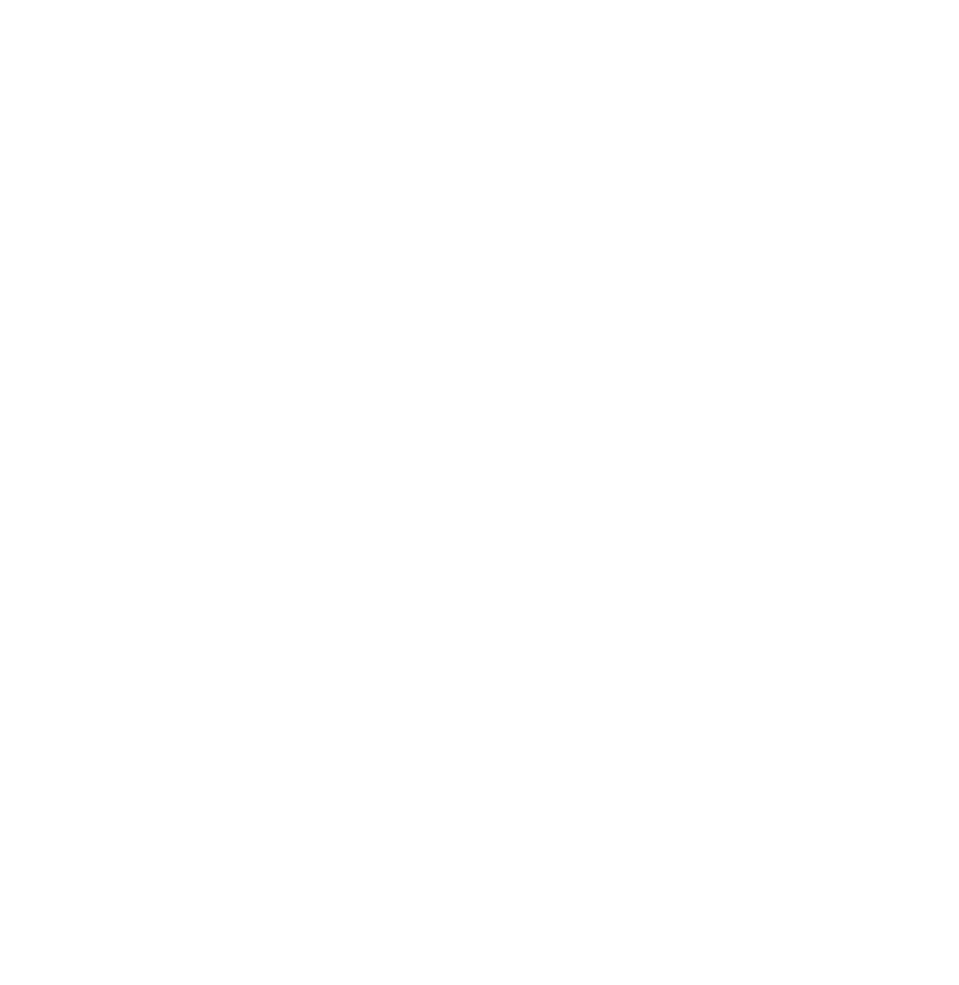

